\title{
Stability of CFL cores in Hybrid Stars
}

\author{
G. Pagliara, J. Schaffner-Bielich \\ Institut für Theoretische Physik, Goethe Universität, D-60438, Frankfurt am Main, Germany
}

\begin{abstract}
We study the equation state of strongly interacting quark matter within a NJL-like model in which the chiral condensates and the color superconducting gaps are computed self-consistently as a function of the baryon density. A vector interaction term is added to the Lagrangian in order to render the quark matter equation of state stiffer. For the low density hadronic phase we use a relativistic mean field model. The phase transition to quark matter is computed by a Maxwell construction. We show that stable CFL cores in hybrid stars are possible if the superconducting gap is sufficiently large. Moreover we find stable stellar configurations in which two phase transitions occur, a first transition from hadronic matter to 2SC quark matter and a second transition from 2SC quark matter to CFL quark matter.
\end{abstract}

PACS numbers:

\section{INTRODUCTION}

The possibility that quark matter, and eventually color superconducting quark matter, is present in the center of neutron stars has stimulated many theoretical investigations in the last years both on the modelling of the equation of state (EoS) of quark matter and on the phenomenological signatures of the presence of quark matter in neutron stars [1]. Presently the "state of the art" for the EoS of quark matter is represented by the three-flavor Nambu-Jona-Lasinio (NJL) model in which both the chiral condensates and the diquark condensates are self-consistently computed as a function of the chemical potential and temperature. Chemical equilibrium and charge neutrality (both electric and color charge) conditions necessary to describe neutron star matter are also imposed in this model [2, 3, 4, 5, 6]. Recently, also the effect of a finite neutrino chemical potential has been included [7, 8]. The structure of the QCD phase diagram within this model turns out to be very rich, with many different possible quark phases. One of the most striking feature, on which we will focus here, is the first order phase transition between the two flavor phase, 2SC or normal quark matter depending on the diquark coupling constant, and the three-flavor superconducting phase, the Color-Flavor-Locking phase (CFL), at vanishing temperature.

Concerning the phenomenological signatures, the differences between the mass-radius relation for neutron stars and quark or hybrid stars is currently studied. The astrophysical data so far are still affected by large uncertainties but will improve considerably with the advent of new satellite missions, as XEUS, Constellation-X, SKA, JWST and LISA. From recent theoretical studies it turns out that the values of the maximum mass of neutron stars and compact stars containing quark matter are very similar [9, 10]. Therefore, it seems difficult with the present knowledge to rule out quark matter from just a mass measurement [11, 12]. Other interesting quantities have been calculated (see Ref. 13 for a recent review) for the different possible phases of quark matter, as the neutrino emissivity and the heat capacity which are important for the cooling of compact stars [14, 15, 16, 17, 18] or the bulk viscosity which determines stability with respect to gravitational waves emission via r-modes [19, 20, 21, 22, 23, 24]. Also in explosive phenomena, as supernovae and gamma-ray-bursts (GRBs), quark matter can play an important role. For instance, the possibility of a double phase transition, first from hadronic matter to 2-flavor quark matter and then from 2-flavor quark matter to the CFL phase has been proposed to explain the complicated time structure of GRBs exhibiting a long quiescent time in their light curve [25, 26, 27].

When the above mentioned NJL-EoS is used for the applications on compact stars, hybrid stars become unstable at the onset of the CFL phase and therefore CFL phase can not be present in the core of neutron stars. This conclusion was obtained first in Ref. 28] where quark matter does not occur at all in compact stars because there is a direct transition from hadronic matter to CFL matter in the model used. In Refs. 29, 30, 31] for different EoSs for the hadronic matter and different parameters for the NJL model for quark matter, again the CFL phase was ruled out because it renders the stars unstable. The 2SC phase could appear; the conclusion is therefore that only 2-flavor superconducting quark matter can be realized in compact stars. A similar result about the allowed quark phases in compact stars was also found in Ref. [32] where a modified 2-flavor NJL model, which simulates confinement at low density, is proposed. The dependence of the stability of a quark core from the momentum cut-off of the NJL model has been analysed using density dependent cut-offs, but the instability still persists [33] 61] .

On the other hand, completely different results are obtained using MIT-bag-like models as shown in Refs. 34, 35, 36. where the appearance of CFL cores does not compromise the stability of the star. Moreover, the absence of the 2SC phase in compact stars has been demonstrated in Ref. [37].

In this paper we want to consider again the NJL-EoS as computed in Ref. [3] to study the structure and composition 
of compact stars. We will investigate larger windows of the model parameters with respect to previous work, with particular attention to the diquark coupling. Furthermore, we discuss the importance of the procedure used to fix the effective bag constant within the NJL model for the stability of a star when the phase transition to quark matter is considered. We will investigate a new procedure for fixing the effective bag constant by requiring that the chiral symmetry restoration coincides with the transition from the hadronic to the quark matter description 62]. We use then different EoSs to compute the mass-radius relations of hybrid stars showing that in some cases a stable CFL core is possible and, even more intriguing, that two phase transitions, from hadronic matter to the 2SC and then to the CFL phase, can take place in compact stars. The double phase transition is particular intriguing in connection with the deconfinement quark model of GRBs in which the interpretation of bursts presenting two emission periods is due to a double phase transition in compact stars [25, 26, 27].

The paper is organised as follows. In Sec. II we discuss a simple toy model for a first order phase transition between hadronic matter and quark matter in compact stars and we study the stability of the star by varying the parameters of the quark matter EoS. In Sec. III we compute the quark matter EoS within the NJL model for different sets of parameters and finally in Sec. IV we discuss the stability of the stars obtained using our EoSs with particular emphasis on the CFL core stability. In Sec. V we draw our conclusions.

\section{A TOY-MODEL FOR PHASE TRANSITIONS IN COMPACT STARS}

We present in this section a toy-model EoS for strongly interacting matter to show qualitatively the conditions for stable solutions of the Tolman-Oppenheimer-Volkoff (TOV) equation when a phase transition from hadronic matter to an exotic phase, like quark matter, occurs. At very low baryon densities, $n \lesssim 0.05 \mathrm{fm}^{-3}$, we use the EoS as computed in Ref. [38] (similar results are obtained using the EoS of Ref. [39]) suitable for the crust of neutron stars. At larger densities, we consider for the hadronic matter a relativistic mean field EoS, GM3, taken from Ref. [40]. For quark matter we adopt a schematic EoS in which the pressure is proportional to the energy density $p=a \epsilon$ where the slope (which corresponds to the sound velocity and therefore regulates the stiffness of the quark matter EoS) is a free parameter. Notice that this EoS corresponds, for $a=1 / 3$, to the EoS of massless and non-interacting quarks. We then model the phase transition from hadronic matter to quark matter by introducing two other free parameters, the energy density jump $\Delta_{\epsilon}=\epsilon_{2}-\epsilon_{1}$ at the onset of the phase transition $\left(\epsilon_{2}\right.$ and $\epsilon_{1}$ are the energy densities of the quark phase and of the hadronic phase) and the pressure at which the phase transition occurs $p_{0}$. Our aim is to investigate, for the different values of these parameters, the stability of stars having a core of quark matter. Let $P 1$ be the parameter set with $a=1 / 3, \Delta_{\epsilon}=200 \mathrm{MeV} / \mathrm{fm}^{3}$ and $p_{0}=200 \mathrm{MeV} / \mathrm{fm}^{3}$. In panel (a) of Fig. 1 the corresponding $\mathrm{EoS}$ is plotted by a gray line. In this case compact stars are unstable when the quark matter phase appears, see the gray line in panel (b). If we decrease now the transition pressure to $p_{0}=50 \mathrm{MeV} / \mathrm{fm}^{3}$ keeping the same values for the other parameters (set $P 2$ see solid line in panel (a)), a sizable branch of stable configurations appears instead, see the thin solid line in panel (b). If we reduce the energy density jump to $\Delta_{\epsilon}=50 \mathrm{MeV} / \mathrm{fm}^{3}$ (set $P 3$ with $p_{0}$ and $a$ as in set $P 1$, see dashed line in panel (c)) a small branch of stable solutions is obtained (dashed line in panel (d)). Concerning the stiffness parameter $a$, to obtain stable solutions one must choose $a=1$ (set $P 4$ with $p_{0}$ and $\Delta_{\epsilon}$ as in $P 1$ ). For this value of $a$ the quark matter EoS is even stiffer than the hadronic matter EoS (see the dotted line in panel (e) for the EoS and the dotted line in panel (f) for the mass-radius relation). This last case is probably not realistic in view of the asymptotic freedom property of QCD.

In conclusion, what seems to be the most crucial parameter for the stability of a quark matter core is the value of the pressure at the onset of the phase transition. If the softening due to the appearance of quark matter occurs at too large pressures, deep in the core of a neutron star, the sourronding material exerts a pressure that can not be sustained by the new formed phase and therefore the star collapses. Also a low value of $\Delta_{\epsilon}$ can help to stabilise the quark matter core. A detailed analytical study on the critical value of energy density jump for having stable cores of a new phase can be found in Refs. [41, 42, 43, 44] demonstrating that $\epsilon_{2} / \epsilon_{1}$ should be not larger than $\epsilon_{2} / \epsilon_{1} \leq \frac{3}{2}\left(1+p_{0} / \epsilon_{1}\right)$.

We will see in the next sections how these cases are connected with more physical meaningful quantities as the bag constant, the superconducting gap and the constituent masses of quarks .

\section{PHASE TRANSITION TO QUARK MATTER}

We present now a more realistic EoS for quark matter. The EoS is computed within the NJL-like model proposed in Ref. [3] in which a scalar diquark interaction term for the color antitriplet and flavor antitriplet channel is added to the usual NJL model. Here we include also the isoscalar vector term as in Ref. [30, 45, 46] in order to obtain stiffer EoSs. The input variables of the model are the chemical potentials for all the quark flavors and colors given, 

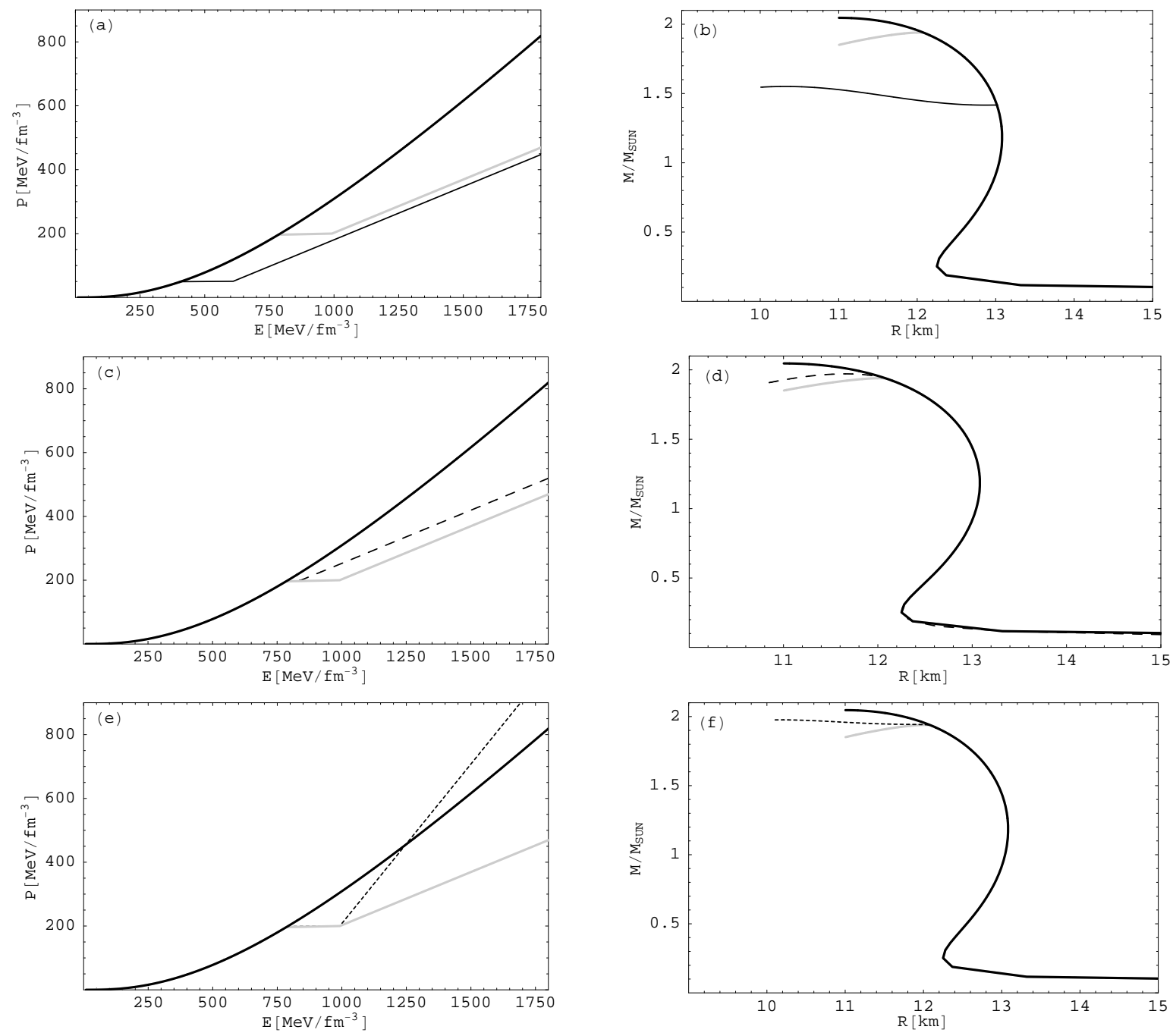

FIG. 1: Equations of state and corresponding mass-radius relations for the toy-model for different parameter sets. EoSs are shown in the left row and mass-radius relations in the right row. The purely hadronic matter EoS and the corresponding neutron stars sequence are depicted by a thick solid line. Panel (a): quark matter EoS for $a=1 / 3, \Delta_{\epsilon}=200 \mathrm{MeV} / \mathrm{fm}^{3}$ and $p_{0}=200 \mathrm{MeV} / \mathrm{fm}^{3}$ (gray line set P1), and $a=1 / 3, \Delta_{\epsilon}=200 \mathrm{MeV} / \mathrm{fm}^{3}$ and $p_{0}=50 \mathrm{MeV} / \mathrm{fm}^{3}$ (solid line set P2). Panel (c): quark matter EoS for the choice $a=1 / 3, \Delta_{\epsilon}=50 \mathrm{MeV} / \mathrm{fm}^{3}$ and $p_{0}=200 \mathrm{MeV} / \mathrm{fm}^{3}$ (dashed line set P3), the other curves are as in panel (a). Panel (e): quark matter EoS for $a=1, \Delta_{\epsilon}=200 \mathrm{MeV} / \mathrm{fm}^{3}$ and $p_{0}=200 \mathrm{MeV} / \mathrm{fm}^{3}$ (dotted line set P4), the other curves as in panel (a). Stable configurations with a quark core can only appear for sufficiently stiff quark matter EoS or for a transition point away from the maximum mass configuration of the purely hadronic compact stars.

in chemical equilibrium, by the matrix:

$$
\mu_{a b}^{\alpha \beta}=\left(\mu \delta^{\alpha \beta}+\mu_{Q} Q_{f}^{\alpha \beta}\right) \delta_{a b}+\left[\mu_{3}\left(T_{3}\right)_{a b}+\mu_{8}\left(T_{8}\right)_{a b}\right] \delta^{\alpha \beta} .
$$

where $\mu$ is the quark chemical potential, $\mu_{Q}$ is the chemical potential of the electric charge equal to minus the electron chemical potential $\mu_{e}, \mu_{3}$ and $\mu_{8}$ are the color chemical potentials associated with the two mutually commuting color charges of the $S U(3)_{c}$ gauge group. The explicit form of the electric charge matrix is $Q_{f}=\operatorname{diag}_{f}\left(\frac{2}{3},-\frac{1}{3},-\frac{1}{3}\right)$, and for the color charge matrices $T_{3}=\operatorname{diag}_{c}\left(\frac{1}{2},-\frac{1}{2}, 0\right)$ and $\sqrt{3} T_{8}=\operatorname{diag}_{c}\left(\frac{1}{2}, \frac{1}{2},-1\right)$.

The model parameters are fixed by fitting low energy hadronic properties which are the current quark masses, the quark-antiquark coupling $G_{S}$, the strength $K$ of the "t Hooft" interaction and the cut-off parameter $\Lambda$ introduced in the NJL model to regularise the ultraviolet divergences:

$$
m_{u, d}=5.5 \mathrm{MeV},
$$



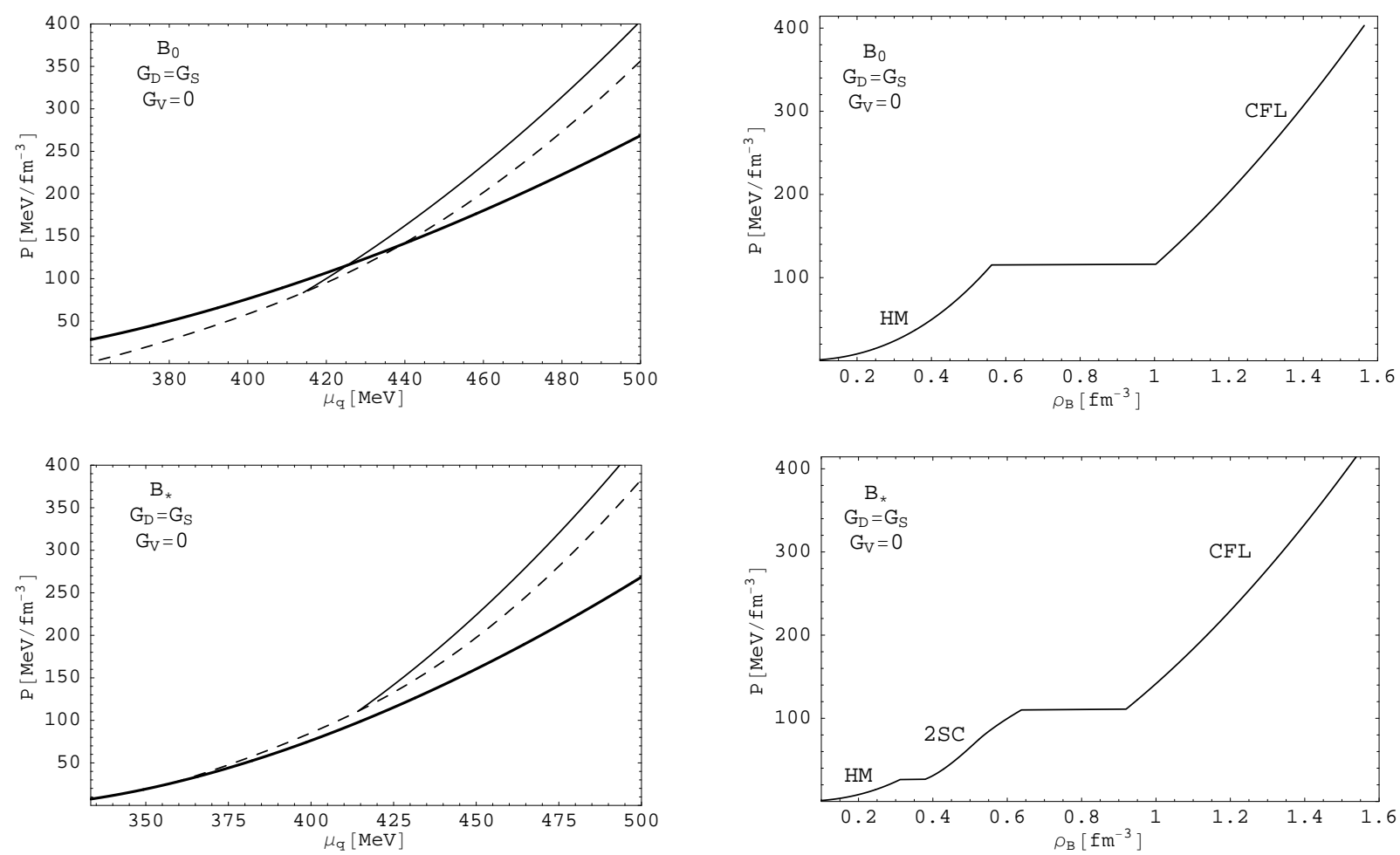

FIG. 2: Upper left panel: pressure as a function of the chemical potential for hadronic matter (thick line), the 2SC phase (dashed line) and the CFL phase (solid line). Upper right panel: pressure as a function of the baryon density. Parameters are: $G_{D}=G_{S}, B=B_{0}, G_{V}=0$. A direct transition from hadronic matter to the CFL phase occurs with a large baryon density jump $\sim 0.45 \mathrm{fm}^{-3}$ at the onset of the phase transition. Lower left panel: pressure as a function of the chemical potential. Lower right panel: pressure as a function of the baryon density. Parameters are: $G_{D}=G_{S}, B=B_{*}, G_{V}=0$. For a lower value of $B$ there is first a transition from hadronic matter to $2 \mathrm{SC}$ quark matter and then from $2 \mathrm{SC}$ quark matter to CFL quark matter.

$$
\begin{aligned}
m_{s} & =140.7 \mathrm{MeV}, \\
G_{S} \Lambda^{2} & =1.835 \\
K \Lambda^{5} & =12.36 \\
\Lambda & =602.3 \mathrm{MeV} .
\end{aligned}
$$

After fixing the masses of the up and down quarks by equal values, $m_{u, d}=5.5 \mathrm{MeV}$, the other four parameters are chosen to reproduce the following four observables [47]: $m_{\pi}=135.0 \mathrm{MeV}, m_{K}=497.7 \mathrm{MeV}, m_{\eta^{\prime}}=957.8 \mathrm{MeV}$, and $f_{\pi}=92.4 \mathrm{MeV}$. This parameter set gives $m_{\eta}=514.8 \mathrm{MeV}[47]$.

There are two more parameters, the diquark coupling $G_{D}$ and the vector current coupling $G_{V}$ which are not known. We will use $G_{D}=G_{S}$ and $G_{D}=1.2 G_{S}$ because one expects that the diquark coupling has the strength as the quark-antiquark coupling. For $G_{V}$ we choose the cases $G_{V}=0$ and $G_{V}=0.2 G_{S}$.

At vanishing temperature and within the mean field approximation the pressure reads:

$$
p=\frac{1}{2 \pi^{2}} \sum_{i=1}^{18} \int_{0}^{\Lambda} \mathrm{d} k k^{2}\left|\epsilon_{i}\right|+4 K \sigma_{u} \sigma_{d} \sigma_{s}-\frac{1}{4 G_{D}} \sum_{c=1}^{3}\left|\Delta_{c}\right|^{2}-2 G_{S} \sum_{\alpha=1}^{3} \sigma_{\alpha}^{2}+\frac{\omega_{0}^{2}}{4 G_{V}}+p_{e}
$$

where $\epsilon_{i}$ are the dispersion relations as computed in Ref. [3] $\sigma_{u, d, s}$ are the quark-antiquark condensates and $\Delta_{c}$ are the three diquark condensates. We denote with $\omega_{0}=2 G_{V}\left\langle Q M\left|\psi_{u}^{\dagger} \psi_{u}+\psi_{d}^{\dagger} \psi_{d}+\psi_{s}^{\dagger} \psi_{s}\right| Q M\right\rangle$ the mean field expectation value of the scalar vector meson $\omega$. This field modify also the chemical potentials: $\mu_{u, d, s} \rightarrow \mu_{u, d, s}-\omega_{0}$. Finally, the contribution to the pressure of electrons is $p_{e}=\mu_{e}^{4} /\left(12 \pi^{2}\right)$.

The pressure within the NJL model is defined but for a constant $B$, similarly to the MIT bag constant, which is usually fixed by the following procedure: one requires that the corrected pressure $p-B$ is vanishing at vanishing chemical potential [4, 48, 49]. In our model, for the parameters set used here, we have $B=B_{0}=(425.4 \mathrm{MeV})^{4}[63]$. 
Actually, this procedure to determine the bag constant is somehow unsatisfying, as also stated in Ref. [49], since the pressure computed within the NJL model at vanishing density is used i.e. in a regime where NJL model can not be trusted due to its lack of confinement. On the other hand, in the MIT bag model for instance, which contains confinement, the pressure in the vacuum is not vanishing.

Here we propose an alternative procedure to fix the bag constant in the NJL model. First, we introduce at low density an EoS having hadronic degrees of freedom, like the GM3 EoS used in Sec. II, and then we compute the transition to quark matter, the deconfinement transition, by a Maxwell construction. We remark that "deconfinement" in our scheme has the meaning of a change of degrees of freedom and the corresponding Lagrangian, it is not a phase transition described by an order parameter. To fix the bag constant we assume that deconfinement occurs at the same chemical potential as the chiral phase transition i.e. when chiral symmetry is restored. Practically this means that we require that the pressure of quark matter, $p-B$, is equal to the pressure of the hadronic matter at the critical chemical potential for which chiral symmetry is restored i.e. the value $\mu_{\text {crit }}$ computed in the NJL model. This allows to fix the value of $B$ and, as we will see, to obtain significantly different results with respect to the ones obtained using the conventional procedure. It turns out that deconfinement occurs at very large quark chemical potential $\sim 470 \mathrm{MeV}$ by far larger than the critical chemical potential for chiral symmetry restoration for $B=B_{0}$, the standard choice [49]. The bag value obtained with our assumption, $B=B_{*}$, as we will see in the following is marginally smaller than $B_{0}$ and must be considered as the lowest possible value for the bag constant in the NJL model because it allows to use the NJL-EoS just starting from $\mu_{\text {crit }}$. For chemical potentials lower than $\mu_{\text {crit }}$ the density of quarks, as computed within the NJL model, is vanishing due to the completely broken chiral symmetry. This is obviously a regime in which the NJL model can not be applied. Our assumption on the coincidence of deconfinement and the chiral phase transitions at finite density, has not yet a QCD-motivated argument. Nevertheless, this coincidence has been found in Lattice QCD calculations at finite temperature (see Ref. [50] and references therein) and it has been also adopted in other models for the EoS at finite chemical potential as the NJL-inspired model proposed in Ref. 32]. Interestingly, within the Dyson-Schwinger approach, it is possible to define an order parameter for deconfinement at finite density and it turns out that the two phase transitions occur simultaneously [51]. We will examine here both choices for the bag constant $B_{0}$ and $B_{*}$.

We remark that another possible scenario has been proposed for the finite density phase transition in Refs. 52, 53]: there is no deconfinement at all at large density, only the chiral phase transition occurs and the quarks are still confined.

\section{A. Results}

In order to compute the EoS needed for compact stars, the pressure, Eq. (7), must be minimised with respect to the chiral and color superconducting order parameters, $\sigma_{\alpha}$ and $\Delta_{c}$, and therefore six gap equations are obtained:

$$
\begin{aligned}
& \frac{\partial p}{\partial \sigma_{\alpha}}=0, \\
& \frac{\partial p}{\partial \Delta_{c}}=0 .
\end{aligned}
$$

Moreover, local electric and color charge neutrality are met if three other three equations are satisfied,

$$
\begin{aligned}
n_{Q} & \equiv \frac{\partial p}{\partial \mu_{Q}}=0 \\
n_{3} & \equiv \frac{\partial p}{\partial \mu_{3}}=0 \\
n_{8} & \equiv \frac{\partial p}{\partial \mu_{8}}=0
\end{aligned}
$$

These conditions fix the values of the three corresponding chemical potentials, $\mu_{Q}, \mu_{3}$ and $\mu_{8}$. One more equation is imposed to compute the vector current expectation value:

$$
\frac{\partial p}{\partial \omega_{0}}=0 .
$$



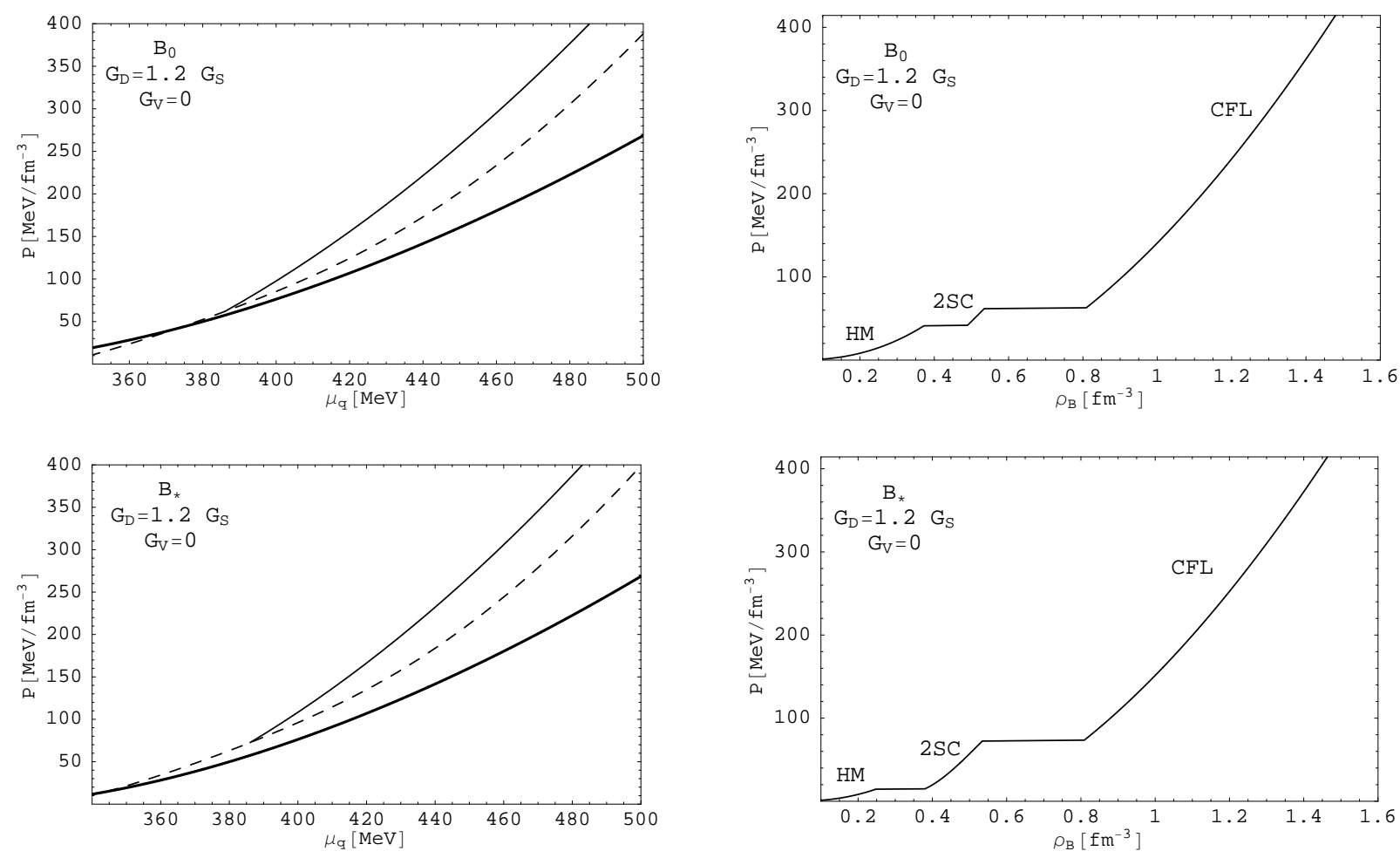

FIG. 3: Same as in Fig. 2 for $G_{D}=1.2 G_{S}$. When a large diquark coupling $G_{D}$ is considered there are for both values of $B$ two phase transitions, first from hadronic matter to 2SC quark matter and then from 2SC quark matter to CFL quark matter.

After all these quantities are fixed, one arrives at the pressure of quark matter as a function of the quark chemical potential only and one can easily build the Maxwell construction by solving the equation:

$$
p_{H M}(\mu)=p_{Q M}(\mu)-B .
$$

As discussed before we use for $B$ two values, $B_{0}$ and $B_{*}$. In the upper left panel of Fig. 2 we show the pressure as function of the quark chemical potential for hadronic matter (thick line) and quark matter (thin line, the dashed line corresponding to the $2 \mathrm{SC}$ phase and the continuous line to the CFL phase). Parameters are $B=B_{0}, G_{D}=G_{S}$ and $G_{V}=0$. In this case there is a direct transition from hadronic matter to the CFL phase, see also Refs. [28, 29]. The corresponding EoS is shown in the right panel of Fig. 2. Notice the large jump of the baryon density of about $\sim 0.45$ $\mathrm{fm}^{-3}$ at the onset of the phase transition. We notice that in Ref. 30] a similar parameter set, $B=B_{0}, G_{D}=G S$ and $G_{V}=0$, gives a different result: there is just a transition from hadronic matter to the 2SC phase. Apart from the different choice for the hadronic matter EoS, the 't Hooft interaction term is neglected in that calculation. We obtain the same result, a transition from hadronic matter to the $2 \mathrm{SC}$ phase, by choosing $K=0$ in our model. The 't Hooft term in fact, as observed in Refs. 47, 54], pushes the 2SC-CFL phase transition to lower chemical potentials rendering the CFL phase the favored quark matter phase also at intermediate densities.

Let us study how this result changes if we choose $B=B_{*}$, which for this set of parameters is $B_{*}=(424.8 \mathrm{MeV})^{4}$, slightly smaller than $B_{0}=(425.4 \mathrm{MeV})^{4}$. By construction now there is first a transition from the hadronic matter to the 2SC matter and then a second transition from 2SC matter to CFL matter, see the corresponding plots in the lower panel of Fig. 2

We repeated the previous calculation for $G_{D}=1.2 G_{S}$. For this value of the diquark coupling the superconducting gap within the CFL phase at $\mu=500 \mathrm{MeV}$ is $\Delta_{C F L} \sim 160 \mathrm{MeV}$. Notice that our present knowledge of the CFL gap concerns only its order of magnitude i.e. $\sim 100 \mathrm{MeV}$; therefore, a CFL gap larger than $100 \mathrm{MeV}$ is not excluded and has been considered also in previous papers [4, 34, 55, 56]. The effect of increasing the diquark coupling on the EoS is to decrease the onset of chiral symmetry restoration, from $\mu_{q}=358 \mathrm{MeV}$ for $G_{D}=G_{S}$ to $\mu_{q}=344 \mathrm{MeV}$ for $G_{D}=1.2 G_{S}$ and the 2SC-CFL phase transition onset, from $\mu_{q}=415 \mathrm{MeV}$ for $G_{D}=G_{S}$ to $\mu_{q}=386 \mathrm{MeV}$ for $G_{D}=1.2 G_{S}$. As shown in Fig. 3 for both choices of $B$ there is a double phase transition with increasing baryon density [64].

We include now in our calculation the vector meson term and set $G_{V}=0.2 G_{S}$ (we consider now only $G_{D}=1.2 G_{S}$ ). The physical effect of this term is a repulsive interaction between quarks which renders the quark matter EoS stiffer. 

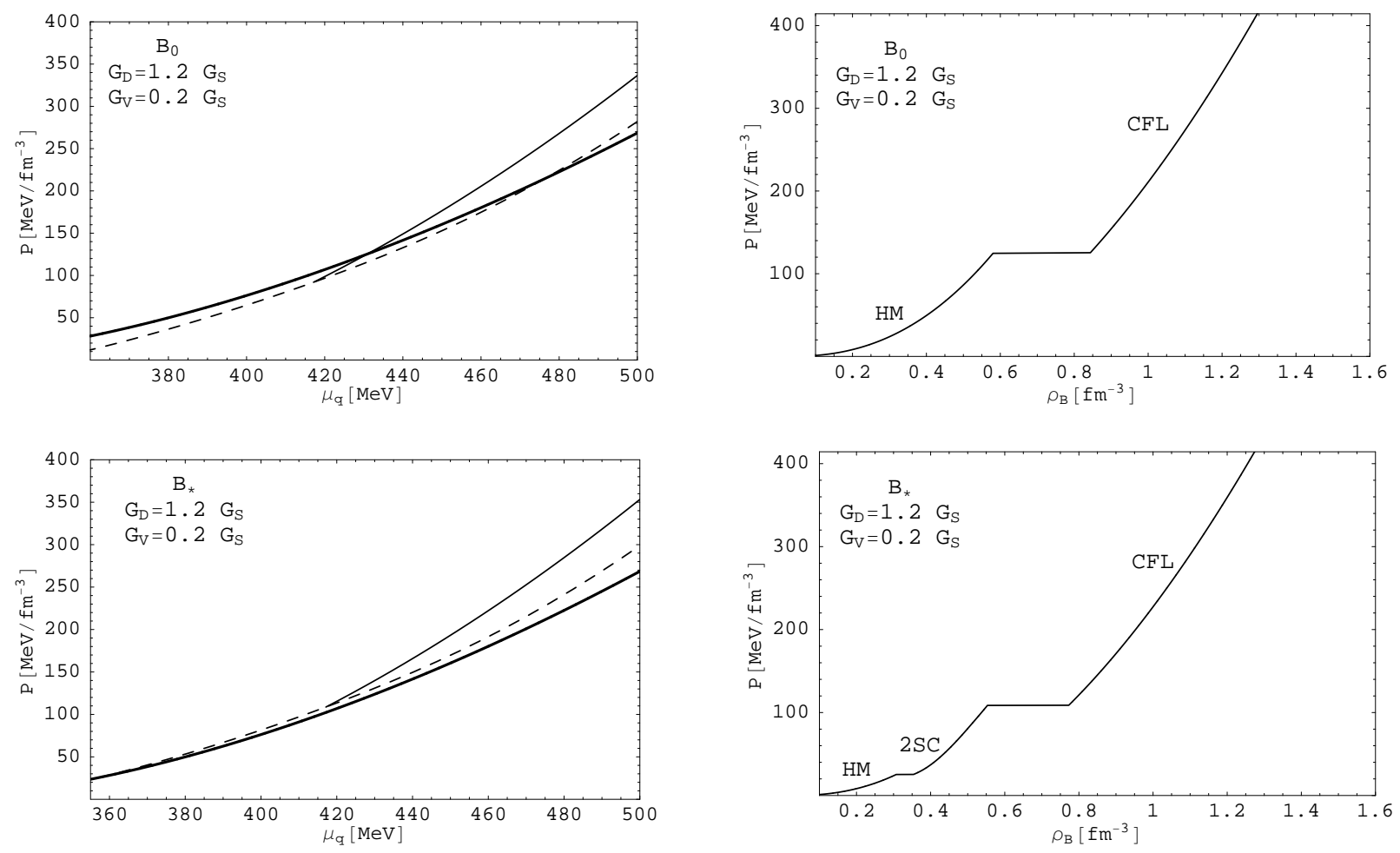

FIG. 4: Same as in Fig. 3 with the inclusion of a repulsive vector term with $G_{V}=0.2 G_{S}$. In the case $B=B_{0}$ a direct transition from hadronic matter to CFL matter is found. In the case $B=B_{*}$ the EoS exhibits instead two phase transitions.

For the case $B=B_{0}$, there is a transition from hadronic matter to CFL matter and for the case $B=B_{*}$ a double phase transition is present, see Fig. (4)

\section{MASS-RADIUS RELATIONS}

Let us now discuss the corresponding mass-radius relations for compact stars. In Fig. [5 we show the mass-radius diagram for the EoSs with $G_{D}=G_{S}$ for $B=B_{0}$ (EoS1) and $B=B_{*}$ (EoS2) indicated by the dotted lines and the EoSs with $G_{D}=1.2 G_{S}$ for $B=B_{0}$ (EoS3) and $B=B_{*}$ (EoS4) indicated by the solid lines. In the first case, EoS1, where a transition from hadronic matter to CFL is found (see upper panel of Fig. 2), the CFL core is unstable and therefore the conclusion is that quark matter does not occur at all in compact stars in agreement with the findings of Ref. [29]. This is due to the fact that the transition to CFL matter occurs at a large pressure and chemical potential which, as demonstrated within the toy model of Sec. I, strongly disfavours stable configurations. Decreasing the value of $B$ to $B_{*}$, which in the toy model would correspond to a change of the transition pressure, changes the result significantly: a stable core of quark matter can be present but only in the $2 \mathrm{SC}$ phase, the subsequent transition to the CFL phase renders the star unstable. This scenario agrees with the one proposed for instance in Ref. [30]. Let us discuss now the cases in which $G_{D}=1.2 G_{S}$. Obviously the larger the diquark coupling the more favoured is quark matter with respect to hadronic matter because both the onset of the chiral symmetry restoration and the one for the 2SC-CFL transition are shifted to lower densities. This implies that the pressure of the phase transition is smaller than in the previous cases but also that the jump in density due to the Maxwell construction is larger [65] . Among these two effects, the first favors the stability of the star while the second disfavors the stability of the star (see our toy-model discussion). We find that the first effect dominates and hybrid stars containing both the 2SC phase and the CFL phase are stable in both cases, $B=B_{0}$ and $B=B_{*}[\underline{66}$. The structure of these stellar objects is extremely interesting: it contains a crust of hadronic matter matter, a layer of 2SC phase and a core of CFL phase. Possible astrophysical implications of these stellar compositions have been already discussed in Refs. 25, 26, 27] in connection with GRBs. The formation of first the 2SC phase and then of the CFL phase, during the evolution of the star, would produce two separate neutrino emission and possibly also two different GRBs emission periods for which there are already observational hints [26].

We point out that the solutions of the TOV equations obtained without vector interactions reach a maximum mass 


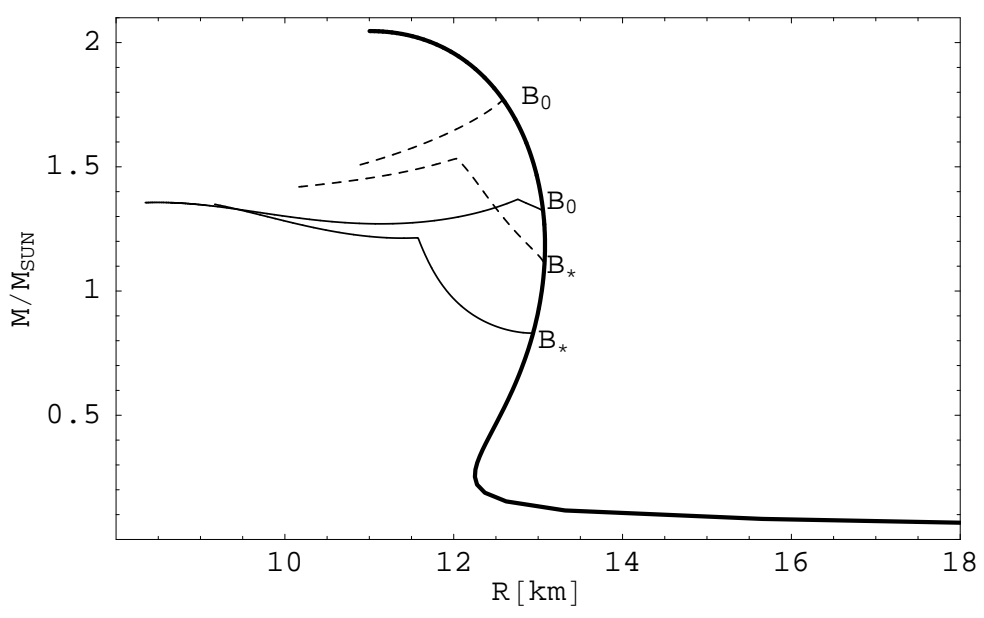

FIG. 5: Mass-radius relations for the EoSs with $G_{D}=G_{S}$ (dashed lines) for $B=B_{0}$ and $B=B_{*}$ and with $G_{D}=1.2 G_{S}$ (solid lines) for $B=B_{0}$ and $B=B_{*}$. $G_{V}=0$ in all cases. The thick curve stands for the hadronic stars. In the case $G_{D}=G_{S}$ no stable CFL cores are found, only a $2 \mathrm{SC}$ core is possible for $B=B_{*}$. In the case $G_{D}=1.2 G_{S}$, hybrid stars having a CFL core are stable. Furthermore, in both cases, $B=B_{0}$ and $B=B_{*}$ a layer of 2SC phase is present inside the hybrid stars.

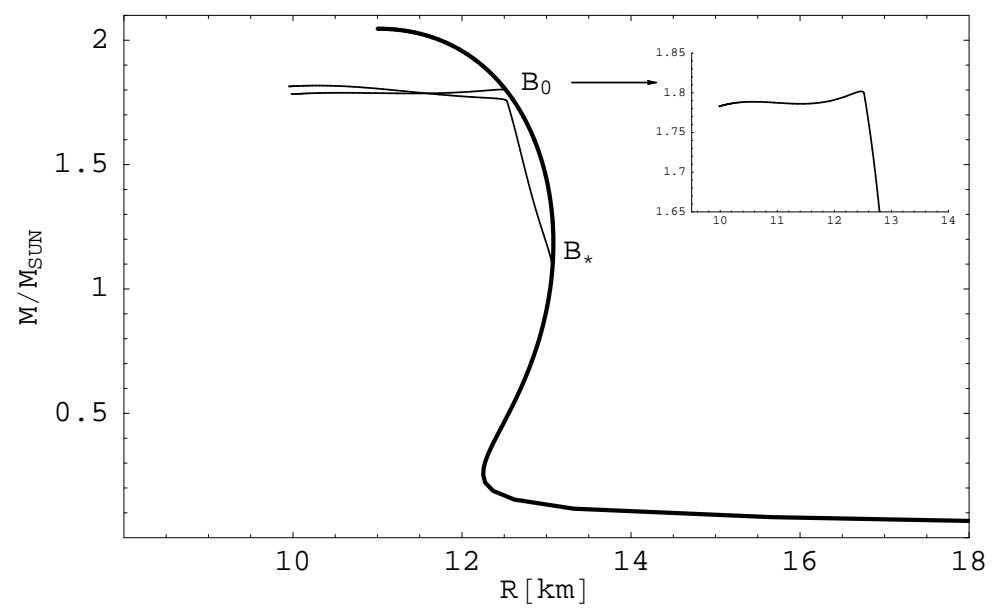

FIG. 6: Mass-radius relations for the EoSs with $G_{D}=1.2 G_{S}$ and $G_{V}=0.2 G_{S}$ for $B=B_{0}$ and $B=B_{*}$ (solid lines). The thick curve stands for the hadronic stars. In both cases stars having a CFL core are stable (see the magnification for the case $\left.B=B_{0}\right)$, in the case $B=B_{*}$ a double phase transition is present.

of less than $1.4 M_{\odot}$ and therefore are excluded by pulsar mass data. For the case $G_{V}=0.2 G_{S}$ we obtain hybrid stars with a stable core of CFL phase in the case $B=B_{0}$ and hybrid stars with both the 2SC and the CFL phases for $B=B_{*}$ as in the previous cases. These solutions have a corresponding maximum mass of $\sim 1.8 M_{\odot}$ (see Fig. 6) due to the effect of the repulsion given by the vector interaction and are not ruled out by the presently available astrophysical data. For even larger values of $G_{V}$, we arrive at stable hybrid stars with just a CFL core.

A final important remark concerns the large value of the diquark coupling that we must use to obtain stable quark matter cores. The crucial quantity for the stability is a low value of the pressure of the phase transition to quark matter as seen already on our discussion of toy-model. A low transition pressure can be obtained for a large diquark coupling but also for a reduced constituent mass of the strange quark. Within the NJL model discussed here, $m_{s}$ turns out to be quite large. Other quark matter studies within the Dyson-Schwinger approach point towards lower values of $m_{s}$ and consequently the CFL phase dominates in an enlarged density region [57]. Unfortunately, the EoS of quark matter within the Dyson-Schwinger approach is not yet available. Our analysis suggests that the corresponding results for the TOV solutions can be very similar to the ones obtained here. 


\section{CONCLUSIONS}

We have studied the phase transition from hadronic matter to quark matter and the corresponding impact on the mass-radius relation of compact stars. By using first a toy-model for the quark matter equation of state we have analysed how the stability of hybrid stars depends on the properties of the phase transition. In particular a low value of the pressure at the onset of the phase transition seems to be the most crucial quantity for the stability of the hybrid star. We have then computed the equation of state of quark matter within the NJL model by including effects from the chiral condensates, the diquark coupling pattern and a repulsion vector term. For large enough values of the diquark coupling strength, hybrid stars containing a CFL core are found to be stable. Moreover, exciting stable hybrid stars containing both a layer of a 2SC phase and a core of a CFL phase appears to be possible. This result opens the possibility to find new signatures of the presence of quark matter in compact stars. The formation of different quark phases in compact stars can release a huge amount of energy powering both energetic bursts of neutrino and gamma-ray signals. A detailed study of the time evolution of a proto-neutron star including the possible appearance of the 2SC phase followed by the formation of the CFL phase could reveal some new features and opportunities for detecting the chiral phase transition of QCD in the sky.

\section{Acknowledgements:}

We thank S. Rüster and D. Rischke for providing us the code to compute the equation of state of quark matter. G.P. thanks M. Buballa and D. Nickel for useful discussions and the INFN for financial support.

[1] M. G. Alford, K. Rajagopal, T. Schaefer, and A. Schmitt (2007), arXiv:0709.4635 [hep-ph].

[2] H. Abuki, M. Kitazawa, and T. Kunihiro, Phys. Lett. B615, 102 (2005), hep-ph/0412382.

[3] S. B. Ruester, V. Werth, M. Buballa, I. A. Shovkovy, and D. H. Rischke, Phys. Rev. D72, 034004 (2005), hep-ph/0503184.

[4] D. Blaschke, S. Fredriksson, H. Grigorian, A. M. Oztas, and F. Sandin, Phys. Rev. D72, 065020 (2005), hep-ph/0503194.

[5] N. D. Ippolito, G. Nardulli, and M. Ruggieri, JHEP 04, 036 (2007), hep-ph/0701113.

[6] A. W. Steiner, S. Reddy, and M. Prakash, Phys. Rev. D66, 094007 (2002), hep-ph/0205201.

[7] S. B. Ruester, V. Werth, M. Buballa, I. A. Shovkovy, and D. H. Rischke, Phys. Rev. D73, 034025 (2006), hep-ph/0509073.

[8] F. Sandin and D. Blaschke, Phys. Rev. D75, 125013 (2007), astro-ph/0701772.

[9] M. Alford, M. Braby, M. W. Paris, and S. Reddy, Astrophys. J. 629, 969 (2005), nucl-th/0411016.

[10] M. Alford et al., Nature 445, E7 (2007), astro-ph/0606524.

[11] F. Ozel, Nature 441, 1115 (2006).

[12] D. J. Nice et al., Astrophys. J. 634, 1242 (2005), astro-ph/0508050.

[13] J. Schaffner-Bielich (2007), arXiv:0709.1043 [astro-ph].

[14] S. Popov, H. Grigorian, and D. Blaschke, Phys. Rev. C74, 025803 (2006), nucl-th/0512098.

[15] I. A. Shovkovy and P. J. Ellis, Phys. Rev. C66, 015802 (2002), hep-ph/0204132.

[16] S. Reddy, M. Sadzikowski, and M. Tachibana, Nucl. Phys. A714, 337 (2003), nucl-th/0203011.

[17] D. N. Aguilera, D. Blaschke, M. Buballa, and V. L. Yudichev, Phys. Rev. D72, 034008 (2005), hep-ph/0503288.

[18] R. Anglani, G. Nardulli, M. Ruggieri, and M. Mannarelli, Phys. Rev. D74, 074005 (2006), hep-ph/0607341.

[19] A. Drago, G. Pagliara, and I. Parenti (2007), arXiv:0704.1510 [astro-ph].

[20] J. Madsen, Phys. Rev. Lett. 85, 10 (2000), astro-ph/9912418.

[21] B. A. Sa'd, I. A. Shovkovy, and D. H. Rischke, Phys. Rev. D75, 065016 (2007), astro-ph/0607643.

[22] M. G. Alford and A. Schmitt, J. Phys. G34, 67 (2007), nucl-th/0608019.

[23] M. G. Alford, M. Braby, S. Reddy, and T. Schafer, Phys. Rev. C75, 055209 (2007), nucl-th/0701067.

[24] A. Drago, A. Lavagno, and G. Pagliara, Phys. Rev. D71, 103004 (2005), astro-ph/0312009.

[25] A. Drago, A. Lavagno, and G. Pagliara, Nucl. Phys. A774, 823 (2006), astro-ph/0510018.

[26] A. Drago and G. Pagliara, Astrophys.J 665, 1227 (2007), astro-ph/0512602.

[27] A. Drago, A. Lavagno, and I. Parenti, Astrophys.J 659, 1519 (2007).

[28] M. Baldo et al., Phys. Lett. B562, 153 (2003), nucl-th/0212096.

[29] M. Buballa, F. Neumann, M. Oertel, and I. Shovkovy, Phys. Lett. B595, 36 (2004), nucl-th/0312078.

[30] T. Klahn et al. (2006), nucl-th/0609067.

[31] A. G. Grunfeld et al. (2007), arXiv:0705.3787 [hep-ph].

[32] S. Lawley, W. Bentz, and A. W. Thomas, J. Phys. G32, 667 (2006), nucl-th/0602014.

[33] M. Baldo, G. F. Burgio, P. Castorina, S. Plumari, and D. Zappala, Phys. Rev. C75, 035804 (2007), hep-ph/0607343.

[34] M. Alford and S. Reddy, Phys. Rev. D67, 074024 (2003), nucl-th/0211046.

[35] A. Drago, A. Lavagno, and G. Pagliara, Phys. Rev. D69, 057505 (2004), nucl-th/0401052.

[36] I. Bombaci, G. Lugones, and I. Vidana, Astron. Astrophys. 462, 1017 (2007), astro-ph/0603644.

[37] M. Alford and K. Rajagopal, JHEP 06, 031 (2002), hep-ph/0204001.

[38] S. B. Ruester, M. Hempel, and J. Schaffner-Bielich, Phys. Rev. C73, 035804 (2006), astro-ph/0509325. 
[39] G. Baym, C. Pethick, and P. Sutherland, Astrophys. J. 170, 299 (1971).

[40] N. K. Glendenning and S. A. Moszkowski, Phys. Rev. Lett. 67, 2414 (1991).

[41] Z. F. Seidov, Ast. Zh. 48, 443 (1971).

[42] J. L. Zdunik, P. Haensel, and R. Schaeffer, Astron. Astrophys. 172, 95 (1987).

[43] L. Lindblom, Phys. Rev. D58, 024008 (1998), gr-qc/9802072.

[44] B. Kaempfer, Physics Letters B 101, 366 (1981).

[45] M. Hanauske, L. M. Satarov, I. N. Mishustin, H. Stoecker, and W. Greiner, Phys. Rev. D64, 043005 (2001), astro$\mathrm{ph} / 0101267$.

[46] R. Manka and G. Przybyla, New J. Phys. 4, 14 (2002), nucl-th/0201003.

[47] M. Buballa, Phys. Rept. 407, 205 (2005), hep-ph/0402234.

[48] M. Buballa and M. Oertel, Phys. Lett. B457, 261 (1999), hep-ph/9810529.

[49] K. Schertler, S. Leupold, and J. Schaffner-Bielich, Phys. Rev. C60, 025801 (1999), astro-ph/9901152.

[50] Y. Hatta and K. Fukushima, Phys. Rev. D69, 097502 (2004), hep-ph/0307068.

[51] A. Bender, G. I. Poulis, C. D. Roberts, S. M. Schmidt, and A. W. Thomas, Phys. Lett. B431, 263 (1998), nucl-th/9710069.

[52] E. S. Fraga, R. D. Pisarski, and J. Schaffner-Bielich, Phys. Rev. D63, 121702 (2001), hep-ph/0101143.

[53] L. McLerran and R. D. Pisarski (2007), arXiv:0706.2191 [hep-ph].

[54] A. Drago, G. Pagliara, and J. Schaffner-Bielich (2007), arXiv:0705.4418 [astro-ph].

[55] F. Neumann, M. Buballa, and M. Oertel, Nucl. Phys. A714, 481 (2003), hep-ph/0210078.

[56] M. Kitazawa, D. H. Rischke, and I. A. Shovkovy (2007), arXiv:0709.2235 [hep-ph].

[57] D. Nickel, R. Alkofer, and J. Wambach, Phys. Rev. D74, 114015 (2006), hep-ph/0609198.

[58] N. Ippolito, M. Ruggieri, D. H. Rischke, A. Sedrakian, and F. Weber (2007), arXiv:0710.3874 [astro-ph].

[59] K. Schertler, C. Greiner, J. Schaffner-Bielich, and M. H. Thoma, Nucl. Phys. A677, 463 (2000), astro-ph/0001467.

[60] N. K. Glendenning and C. Kettner, Astron. Astrophys. 353, L9 (2000), astro-ph/9807155.

[61] Very recently, stable hybrid stars configurations have been also obtained by adopting the three flavor LOFF phase for the quark matter EoS [58].

[62] In the following, we adopt the term deconfinement transition for the switch from the hadronic to the quark model, as usually done in the literature. However, the Polyakov loop is not a good order parameter for the deconfinement transition at finite chemical potential anymore but only the quark condensate for the chiral phase transition [52, 53].

[63] To compare with Ref. [49], consider that within our formalism the bag constant corresponds to $B_{0}=B_{0}^{\text {ref }}+$ $\sum_{u, d, s} \frac{3}{\pi^{2}} \int_{0}^{\Lambda} \mathrm{d} k k^{2} \sqrt{k^{2}+m_{i}^{2}}$ where $B_{0}^{r e f}=(217.6 \mathrm{MeV})^{4}$ from Ref. [49]. We have checked that our EoS for normal quark matter is equal to the one presented in that paper.

[64] It is interesting to notice that in the cases in which a transition from hadronic matter to the 2SC phase is found, the two order parameters represented by the chiral condensate and the superconducting gap both abruptly change at the same chemical potential. It is therefore interesting to investigate, in a model independent way, whether the superconducting gap can be considered as the order parameter of confinement at finite density.

[65] Notice that increasing the diquark coupling increases the slope of the pressure as a function of the chemical potential and therefore also the baryon density.

[66] We point out that in the case $B=B_{0}$, the branch of stars containing the CFL phase exhibits first a region of unstable solutions and then stable solutions. These new stable solutions represent stars belonging to the so-called "third family" of compact stars found in Ref. [59, 60]. 